\title{
Über das Verhalten des Atoxyls im Organismus.
}

Von

\section{J. Igersheimer und A. Rothmann.}

(Aus dem pharmakologischen Institut der Universität Heidelberg.)

(Der Redaktion zugegangen am 19. März 1909.)

Die vorliegenden Untersuchungen nahmen ihren Ausgang von klinischen und experimentellen Erfahrungen. Diese lehren, daß das Atoxyl in seinem Intoxikationsablauf zwar manche Ähnlichkeiten mit den anorganischen Arsenverbindungen aufweist, im großen und ganzen aber ein wohlcharakterisiertes, spezifisches Vergiftungsbild hervorruft. Abgesehen von der großen Bedeutung des Atoxyls für die Trypanosomen waren es vor allem 3 Tatsachen:

1. die elektive Wirkung des Atoxyls auf das Sehorgan und ihr von den wenigen ähnlichen Beobachtungen bei Arsenvergiftung abweichender Charakter, ${ }^{1}$ )

2. die ganz spezifische Reaktion des Zentralnervensystems der Katze (Ataxie, Spasmen, spast. Paresen) auf Atoxylvergiftung, ${ }^{2}$ )

3. die das Vergiftungsbild beim Hund völlig beherrschenden und nie fehlenden, starken Hämorrhagien in der Niere, die bei anorganischen Arsen-Intoxikationen sehr selten oder gar nicht beobachtet werden, ${ }^{2}$ )

die uns die Frage aufdrängten, ob eine nähere Erforschung des Schicksals der Verbindung ihre eigenartigen Wirkungen aufzuklären vermag.

Schon seit Bunsen weiß man, daß die organische Kakodylsäure anders wirkt als die arsenige und Arsensäure, jedoch ist aus zahlreichen klinischen und einzelnen experimentellen Beobachtungen über diese aliphatische Arsenverbindung nichts darüber bekannt geworden, daß ähnliche Vergiftungssymptone danach auftraten, wie sie bei der aromatischen Arsenverbindung, dem Atoxyl, zu finden sind. Eine zweite aromatische, von uns untersuchte As-Verbindung, das phenylarsinsaure $\mathrm{Na}$

1) Igersheimer, Ber. über d. XXXV. ophth. Ges. zu Heidelberg 1908. (Ausführliche Mitteilung erscheint demnächst.)

2) Igersheimer, Schmiedeberg-Festschr. d. Arch. f. exp. Path. u. Pharm., 1908, S. 282. (Vorläufige Mitteilung.) 
(eigens von E. Merck hergestellt) rief bei der Katze ganz analoge, nervöse Erscheinungen hervor wie das Atoxyl.

\section{A. Ausscheidung des Atoxyls.}

Als wir mit Untersuchungen über die Ausscheidung des Atoxyls begannen, war aus den Versuchen von Croner und Seligmann ${ }^{1}$ ) bekannt, daß bei einmaliger Verabreichung von Atoxyl in den ersten 4-8 Stunden am meisten As ausgeschieden wird, daß As aber noch bis zu 22 Stunden nachher in Spuren nachweisbar bleibt; bei wiederholter Darreichung von Atoxyl zeigte sich eine viel protrahiertere Ausscheidung von As und dasselbe war auch dann im Kot zu finden. Blumenthal $\left.{ }^{2}\right)$ schloß sich der Anschauung, daß schon einige Stunden nach der Injektion von Atoxyl As im Harn zu finden ist, entgegen seiner eigenen früheren Annahme, an, beharrte aber bei seiner Behauptung, daß die As-Ausscheidung nach einmaliger Einverleibung des Atoxyls nach 24 Stunden noch nicht beendet ist. Auch $\mathrm{Keg} \mathrm{el}^{3}$ ) kommt bei Versuchen an Pferden und Hunden zu ähnlichen Resultaten. Quantitative Versuche führten Croner und Seligmann zu dem Ergebnis, daß von dem mit dem Atoxyl eingeführten As etwa 50-75\% wleder ausgeschieden werden.

Die wichtigste Frage, in welcher Form das Atoxyl den Körper verläßt, war durch die bisherigen Untersuchungen noeh nicht klargestellt. Wir suchten dieser Frage auf quantitativem Wege näher zu kommen und stützten uns dabei auf die von Blumenthal ${ }^{4}$ ) mitgeteilte Beobachtung, daß das Atoxyl im Harn atoxylvergifteter Tiere durch einen bei Zusatz von $\alpha$ Naphthol entstehenden Azofarbstoff nachweisbar ist.

\section{Zur Methode:}

Nachdem wir uns davon überzeugt hatten, daß im Harn atoxylvergifteter Tiere in der Tat ein schöner roter, in Alkali

1) Croner u. Seligmann, D. med. Wochenschr., 1907, Bd. XXV, S. 995 .

2) Blumenthal, Med. Klinik, 1097, Nr. 12, S. 319. D. med. Wochenschrift, 1907, Nr. 26, S. 1065.

3) Kegel, Untersuchungen über die Ausscheid. d. Atoxyls u. d. kakodylsaur. Na im Harn u. Kot. - Inaug.-Diss., Gießen 1908, Sep.-Abdr.

$\left.{ }^{4}\right) \cdot$ Blumenthal, Deutsch. med. Wochenschr., 1908, Nr. 26, S. 1161. 
leicht löslicher Farbstoff durch Kuppelung mit $\alpha$-Naphthol entsteht, gelang es dem einen von uns (R.), auf diese Reaktion eine quantitative kolorimetrische Methode der Atoxylbestimmung $\mathrm{zu}$ gründen. ${ }^{1}$ )

Die Methode besteht darin, daß die Farbintensität der Lösung des Azofarbstoffes in bestimmter Schichtlänge mit einer Farbstofflösung von bekanntem Gehalt an Atoxyl verglichen wird. Wir benutzten zur Vergleichung ein Kolorimeter, das von Gottlieb und Stangassinger ${ }^{2}$ ) angegeben und von ihnen zur quantitativen Bestimmung von Kreatin und Kreatinin benutzt worden ist.

Als Vergleichslösung diente eine frisch bereitete Farbstofflösung von $100 \mathrm{ccm}$, die $20 \mathrm{mg}$ Atoxyl enthält. Aus den verschieden hohen Flüssigkeitssäulen läßt sich dann durch einfache Rechnung der Atoxylgehalt der Untersuchungsflüssigkeit, deren Volumen gleichfalls auf $100 \mathrm{ccm}$ gebracht ist, finden. Bei unserer Bestimmung betrug die Flüssigkeitssäule der Vergleichslösung im Kolorimeter $0,5 \mathrm{~mm}$, bei welcher Höhe man noch eine sehr deutliche Färbung beobachtet. Es ist nicht ratsam, die Flüssigkeitssäule der Vergleichslösung auf $1 \mathrm{~mm}$ oder höher einzustellen, da unter diesen Umständen kleine Veränderungen der Farbnuance sehr schwer wahrnehmbar sind, im Gegenteil war es öfters nötig, die Höhe der Vergleichslösung auf 0,3 oder $0,2 \mathrm{~mm}$ einzustellen, wenn der Gehalt der Untersuchungsflüssigkeit sehr gering war. In diesem Falle wird man auch das Volumen der Untersuchungsflüssigkeit sehr gering bemessen und auf 100 umrechnen. Umgekehrt kann es vorkommen, daß dieselbe sehr viel Atoxyl enthält; hier wird man die Flüssigkeit auf ein höheres Volumen $(200-500 \mathrm{ccm})$ auffüllen und entsprechend auf $100 \mathrm{um}$ rechnen. In jedem Falle wird man vor jeder quantitativen Bestimmung durch einen Reagenzglasversuch feststellen, ob viel oder wenig Atoxyl in der Untersuchungsflüssigkeit $z u$ erwarten ist.

Es sei gleich von vornherein darauf hingewiesen, daß die Einstellung der beiden Gesichtsfelder des Kolorimeters auf gleiche Färbungsintensität eine gewisse Übung erfordert, daß aber diese Übung unschwer $z u$ erlangen ist und dann mit dem Apparat äußerst schnelle und genaue Analysen zu erreichen sind. Wir selbst haben die Vorsicht gebraucht, unabhängig von einander die Werte abzulesen und zu vergleichen. Es ergaben sich fast immer auf $1 / 10 \mathrm{~mm}$ übereinstimmende Zahlen. Wir

1) Unsere Methode war bereits ausgearbeitet, als Covelli in der Chemikerzeitung (Cöthen 1908, S. 1006) darauf hinwies, daß die $\alpha$-Naphtholreaktion eine kolorimetrische Bestimmung des Atoxyls ermögliche.

2) Diese Zeitschrift, Bd. LII, S. 4. 
haben von jeder Untersuchungsflüssigkeit zwei Bestimmungen und zu einer jeden Bestimmung fünf Ablesungen gemacht und hieraus das Mittel genommen.

$\mathrm{Zu}$ unseren Untersuchungen diente ein Präparat, das $5 \mathrm{Mol}$. $\mathrm{H}_{2} \mathrm{O}$ enthielt, wie die Analysen zeigten.

$0,3520 \mathrm{~g}$ Atoxyl verloren $0,0948 \mathrm{~g} \mathrm{H}_{2} \mathrm{O}$ - $0,3000 \mathrm{~g}$ Atoxyl gaben $0,1412 \mathrm{~g} \mathrm{Mg}_{2} \mathrm{As}_{2} \mathrm{O}_{7}$.

Berechnet für $\mathrm{C}_{6} \mathrm{H}_{7} \mathrm{NAsO}_{3} \mathrm{Na}+5 \mathrm{H}_{8} \mathrm{O}: \quad 27,3 \% \mathrm{H}_{2} \mathrm{O} \quad 22,78 \%$ As Gefunden > $>$ > $26,9 \%$, $22,76 \%$,

Die kolorimetrische Bestimmung wurde zuerst an reinen Atoxyllösungen ausgeführt. Die Vergleichslösung wird dargestellt, indem man $20 \mathrm{mg}$ Atoxyl in wenig kaltem Wasser auflöst, mit $1,4 \mathrm{ccm}{ }^{1 / 10-n-H C l ~ v e r s e t z t ~ u n d ~ m i t ~} 0,8 \mathrm{ccm}{ }^{1 / 10}-\mathrm{n}-\mathrm{NaNO}_{\mathbf{2}}-$ lösung diazotiert. Nach einigen Minuten gießt man die Diazolösung in $6,6 \mathrm{ccm} 1 / 100-n-\alpha$-Naphthollösung $(1,44 \mathrm{~g} \alpha$-Naphthol

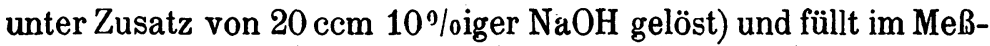
kolben auf $100 \mathrm{ccm}$ auf. Die Vergleichslösung hält sich, wenn an kühlem Ort aufbewahrt, tagelang. In ebensolcher Weise wurde mit den Atoxyllösungen verfahren, die zur Untersuchung dienten, wobei auch entsprechend aliquote Mengen Nitritlösung usw. genommen werden müssen. Die folgende Tabelle I zeigt, daß die Werte des wiedergefundenen Atoxyls um $\pm 2 \%$ schwanken.

Tabelle I.

\begin{tabular}{c|c|c|c}
\hline \multirow{2}{*}{ Nr. } & $\begin{array}{c}\text { Angewandte } \\
\text { Menge Atoxyl } \\
\text { in mg }\end{array}$ & \multicolumn{2}{|c}{ Wiedergefundenes Atoxyl } \\
\cline { 2 - 4 } & in mg & in \% \\
\hline 2 & 30 & 30,08 & 100 \\
3 & 15 & 14,8 & 98,3 \\
4 & 10 & 9,8 & 98 \\
5 & 10 & 10,1 & 101 \\
6 & 5 & 4,9 & 98 \\
7 & 5 & 5,1 & 102 \\
8 & 5 & 5,03 & 100,6 \\
9 & 2 & 1,98 & 99 \\
10 & 2 & 2,04 & 102 \\
& 1 & 1,00 & 100 \\
\end{tabular}


Zur Ausführung der Bestimmung bringt man in den einen Zylinder des Kolorimeters die Vergleichslösung und stellt die gewählte Höhe der Flüssigkeitssäule genau auf $0,5 \mathrm{~mm}$ bezw. 0,3 oder $0,2 \mathrm{~mm}$. In den anderen Zylinder gibt man die gefärbte Untersuchungslösung und bringt durch Heben oder Senken des Zylinders beide Flüssigkeiten auf Farbengleichheit. Die Höhe der dazu erforderlichen Flüssigkeitssäule wird nun abgelesen. Die dem kolorimetrischen Werte entsprechende Menge Atoxyl ist leicht $\mathrm{zu}$ berechnen. Nehmen wir an, das Mittel aus fünf Ablesungen ergebe die kolorimetrischen Werte $2,04 \mathrm{~mm}$, so berechnet sich der Atoxylgehalt bei einer Vergleichslösung von $20 \mathrm{mg}$ Atoxyl und $0,5 \mathrm{~mm}$ Flüssigkeitssäule derselben nach dem Ansatz

$$
\left.20: X=2,04: 0,5 \text { oder } \frac{20 \times 0,5}{2,04}=4,09 \mathrm{mg}^{1}{ }^{1}\right)
$$

Daß man auch bei Zusatz von Atoxyl zu Harn brauchbare Werte erhält, zeigt die folgende Tabelle II.

Tabelle II.

\begin{tabular}{c|c|c|c|c}
\hline $\mathrm{Nr}$. & $\begin{array}{c}\text { Angewandtes } \\
\text { Atoxyl } \\
\text { in mg }\end{array}$ & Harn & \multicolumn{2}{|c}{ Wiedergefundenes Atoxyl } \\
\cline { 3 - 5 } & in ccm & in mg & in $\%$ \\
\hline 1 & 10 & 20 & 10,2 & 102 \\
2 & 10 & 20 & 10,52 & 105 \\
3 & 10 & 10 & 10,05 & 100,5 \\
4 & 10 & 10 & 9,88 & 98,8 \\
5 & 4 & 10 & 4,09 & 102 \\
6 & 4 & 10 & 4,03 & 100,8 \\
7 & 2 & 10 & 1,90 & 95 \\
8 & 10 & 70 & 9,8 & 98 \\
& & & &
\end{tabular}

1) Im Anschluß an die kolorimetrische Bestimmung reiner Atoxyllösung sei erwähnt, daß es auch möglich ist, Arsacetin, die Acetylverbindung des Atoxyls, ebenso zu bestimmen, wie die folgenden Analysen zeigen:

1. $10 \mathrm{mg}$ Arsacetin werden 1 Stunde mit $10 \mathrm{ccm}$-HCl unter Rückfluß gekocht.

Gefunden: $8,17 \mathrm{mg}$ Atoxyl statt $8,87 \mathrm{mg}$ Atoxyl.

2. $10 \mathrm{mg}$ Arsacetin, wie 1 .

$$
(8,87, \quad=10 \mathrm{mg} \text { Arsacetin.) }
$$

Gefunden: $8,85 \mathrm{mg}$ statt $8,87 \mathrm{mg}$ Atoxyl. 
Nachdem so die Zuverlässigkeit der Methode am Harn mit bekanntem Atoxylgehalt festgestellt war, konnten wir dazu übergehen, Harn atoxylvergifteter Versuchstiere auf ausgeschiedene Atoxylmengen $\mathrm{zu}$ untersuchen.

Die Bestimmung von unbekannten Mengen Atoxyl im Harn geschieht folgendermaßen. Eine bestimmte Menge Harn, etwa 10 oder $20 \mathrm{ccm}$, wird mit wenigen Tropfen konzentrierter $\mathrm{HCl}$ schwach kongosauer gemacht und hierauf tropfenweise so lange mit 1/10-n-NaNO ${ }_{2}$-Lösung versetzt, bis ein herausgenommener Tropfen Jodkaliumstärkepapier bläut und diese Bläuung auch nach einigen Minuten noch eintritt. Aus der Menge der zugefügten Nitritlösung berechnet man wie oben angegeben die erforderliche Menge $\alpha$-Naphthollösung (auf $0,4 \mathrm{ccm}$ Nitritlösung kommen $3,3 \mathrm{ccm} \alpha$-Naphthollösung), der man noch ca. $20 \mathrm{ccm} 20 \%$ ige Sodalösung zufügt.

Zur Ergänzung unserer kolorimetrischen Atoxylbestimmungen im Harn wurden Bestimmungen des Gesamtarsens ausgeführt. $\mathrm{Zu}$ diesem $\mathrm{Zweck}$ wurde der Harn eingedampft und durch konzentrierte Salpetersäure und konzentrierte Schwefelsäure vollständig zerstört. ${ }^{1}$ ) Das Arsen wurde durch Schwefelwasserstoff als Sulfid gefällt und in der üblichen Weise teils als Magnesiumpyroarseniat, $\mathrm{Mg}_{8} \mathrm{As}_{8} \mathrm{O}_{7}$, teils als Magnesiumammoniumarseniat, $\mathrm{MgAsO}_{4} \mathrm{NH}_{4} \cdot 1 / 2 \mathrm{H}_{2} \mathrm{O}$ bestimmt. Der Kot wurde getrocknet, zerrieben und in gleicher Weise zerstört, ebenso die Organe. Zur Bestimmung kleiner Arsenmengen bedienten wir uns des von Polenske ${ }^{2}$ ) angegebenen Verfahrens, das auf der Wägung des im Marshschen Apparate erzeugten Arsenspiegels beruht. Wir brauchen wohl kaum noch zu erwähnen, daß wir uns öfters von der Arsenfreiheit der Reagenzien überzeugt haben.

Hier mögen noch einige Gesamtarsenbestimmungen von Harn, dem bekannte Mengen Atoxyl zugesetzt waren, folgen:

1. $100 \mathrm{ccm}$ Harn + 0,2 g Atoxyl gaben $0,0925 \mathrm{~g} \mathrm{Mg}_{2} \mathrm{As}_{2} \mathrm{O}_{7}=0,0447 \mathrm{~g} \mathrm{As}$. die $0,1952 \mathrm{~g}$ Atoxyl d. i. 97,7\% entsprechen.

2. $100 \mathrm{ccm}$ Harn $+0,1 \mathrm{~g}$ Atoxyl gaben $0,0466 \mathrm{~g} \mathrm{Mg}_{2} \mathrm{As}_{2} \mathrm{O}_{7}=0,0225 \mathrm{~g} \mathrm{As}$, die $0,0986 \mathrm{~g}$ Atoxyl, d. i. 98,6\% entsprechen.

(Theoretisch entsprechen $0,0228 \mathrm{~g}$ As $100 \mathrm{mg}$ Atoxyl.)

1) Dragendorff, Ermittlung von Giften, S. 355, Meth. IX.

$\left.{ }^{2}\right)$ Polenske, Arb. a. d. kaiserl. Ges.-Amt, Bd. V, S. 357. Ref. chem. Zentralbl., 1889, Bd. II, S. 58. 
$\mathrm{Zu}$ unseren quantitativen Ausscheidungsversuchen benutzten wir Kaninchen, Katzen und Hunde. Ein Versuch am Menschen, der unten auch näher mitgeteilt werden soll, belehrt uns, daß zwischen Tier und Mensch keine prinzipiellen Unterschiede in der Ausscheidung des Atoxyls im Harn bestehen. Die Tiere erhielten alle eine bestimmte Menge Atoxyl subkutan injiziert. Bei Kaninchen ist es leicht, die Ausscheidung von Stunde $\mathrm{zu}$ Stunde $\mathrm{zu}$ verfolgen. Auch bei den anderen Tieren suchten wir eine Vorstellung von der zeitlichen Ausscheidung des Atoxyls zu gewinnen. Da wir jedoch den Harn nicht durch Katheterisieren abgrenzten, sind wir uns darüber klar, daß die Versuche nur orientierenden Wert haben.

Die folgenden Tabellen III-VIII zeigen die quantitativen Ausscheidungsverhältnisse des Atoxyls im Harn der Versuchstiere sowie des Menschen.

\section{Tabelle III.}

Graues Kaninchen A von $2,250 \mathrm{~kg}$.

\begin{tabular}{|c|c|c|c|c|c|}
\hline \multirow{2}{*}{$\mathrm{Nr}$. } & \multirow{2}{*}{$\begin{array}{l}\text { Harn- } \\
\text { menge } \\
\text { in ccm }\end{array}$} & \multirow{2}{*}{$\begin{array}{c}\text { Stunden } \\
\text { bezw. Tage } \\
\text { nach der } \\
\text { Injektion }\end{array}$} & \multirow{2}{*}{$\begin{array}{c}\text { Aus- } \\
\text { geschiedenes } \\
\text { Atoxyl } \\
\text { in } \mathrm{mg}\end{array}$} & \multicolumn{2}{|c|}{ Gesamtarsen } \\
\hline & & & & in $\mathrm{g} \mathrm{As}$ & $\begin{array}{l}\text { entspr. } \\
\text { Atoxyl } \\
\text { in } \mathrm{mg}\end{array}$ \\
\hline
\end{tabular}

I. Injektion von $50 \mathrm{mg}$ Atoxyl am 9. XI. 08, mittags $12 \mathrm{Uhr}$.

\begin{tabular}{l|r|l|l|l|l|}
1 & 85 & 4 & 20,07 & 0,0098 & 42,98 \\
2 & 40 & $6^{1 / 2}$ & 2,87 & & \\
3 & 47 & 9 & - & - & - \\
4 & 210 & 21 & - & - & - \\
5 & 62 & $24^{1 / 2}$ & - & - & - \\
6 & 91 & $27^{1 / 2}$ & - & - & -
\end{tabular}

II. Injektion von $50 \mathrm{mg}$ Atoxyl am 10. XI. 08, nachmittags $3.45 \mathrm{Uhr}$.
\begin{tabular}{r|r}
7 & 41 \\
8 & 5 \\
9 & 42
\end{tabular}
41
5
42
$21 / 4$
$51 / 4$
$181 / 4$
21,00
4,63
-
$\mid \begin{gathered}0,0089 \\ -\end{gathered}$
39,03 
Tabelle III.

Fortsetzung.

\begin{tabular}{l|c|c|c|c|c}
\hline \hline \multirow{2}{*}{ Nr. } & Harn- & $\begin{array}{c}\text { Stunden } \\
\text { bezw. Tage }\end{array}$ & Aus- & \multicolumn{2}{|c}{ Gesamtarsen } \\
& menge & $\begin{array}{c}\text { geschiedenes } \\
\text { nach der } \\
\text { injektion }\end{array}$ & $\begin{array}{c}\text { Atoxyl } \\
\text { in mg }\end{array}$ & in g As & $\begin{array}{r}\text { entspr. } \\
\text { Atoxyl } \\
\text { in mg }\end{array}$ \\
& in ccm & Injekt & &
\end{tabular}

III. Injektion von $50 \mathrm{mg}$ Atoxyl am 11. XI.08, nachmittags $1 \mathrm{Uhr}$.

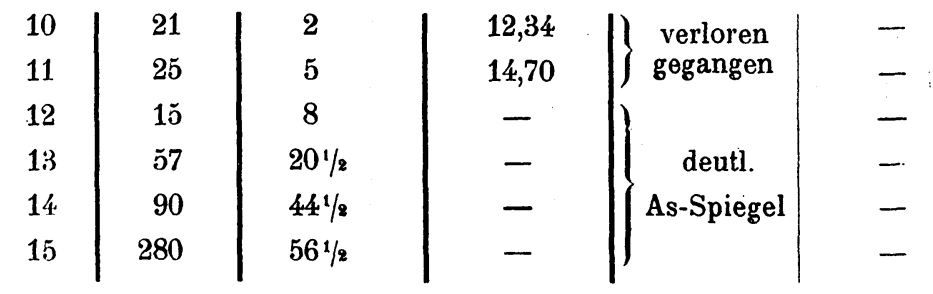

IV. Injektion von $50 \mathrm{mg}$ Atoxyl am 16. XI. 08, nachmittags 4 Uhr. (Harn ist arsenfrei.)

\begin{tabular}{|c|c|c|c|c|}
\hline $\mid 230$ & 24 & 40,24 & 0,0105 & 46,05 \\
\hline $26 \check{5}$ & 48 & - & & \\
\hline 255 & 72 & - & 0,0004 & 1,75 \\
\hline 760 & 7 Tage & - & & \\
\hline 120 & 8 & - & $\begin{array}{c}\text { geringer } \\
\text { As-Spiegel }\end{array}$ & - \\
\hline 140 & 9 & - & & - \\
\hline
\end{tabular}

V. Injektion von $100 \mathrm{mg}$ Atoxyl am 3. XII. 08, vormittags $11 \mathrm{Uhr}$. \begin{tabular}{l|r|rr|c|c|c}
22 & 35 & \multicolumn{2}{|c|}{4} & 84,15 & & \\
23 & 155 & 21 & 10,24 \\
24 & 40 & 45 & - & 0,0220 & 96,07 \\
25 & 580 & 6 & Tage & - & & \\
26 & 190 & 7 & $>$ & - & - & -
\end{tabular}

VI. Injektion von $300 \mathrm{mg}$ Atoxyl am 3. XII. 08, vormittags $11 \mathrm{Uhr}$. (Gewicht des Tieres 1,840 kg.)
27
28
170
24
28
29
170
112
48
30
9 ?
7 Tage
209,86
-
-
-
$\left\{\begin{array}{l}0,0481 \\ 0,0045\end{array}\right.$
212,83
19,82 
Tabelle IV.

Rotes Kaninchen B von $1,670 \mathrm{~kg}$.

\begin{tabular}{|c|c|c|c|c|c|}
\hline \multirow{2}{*}{$\mathrm{Nr}$} & \multirow{2}{*}{$\begin{array}{l}\text { Harn- } \\
\text { menge } \\
\text { in ccm }\end{array}$} & \multirow{2}{*}{$\begin{array}{c}\text { Stunden } \\
\text { bezw. Tage } \\
\text { nach der } \\
\text { Injektion }\end{array}$} & \multirow{2}{*}{$\begin{array}{c}\text { Aus- } \\
\text { geschiedenes } \\
\text { Atoxyl } \\
\text { in mg }\end{array}$} & \multicolumn{2}{|c|}{ Gesamtarsen } \\
\hline & & & & in $\mathrm{g} \mathrm{As}$ & $\begin{array}{l}\text { entspr. } \\
\text { Atoxyl } \\
\text { in } \mathrm{mg}\end{array}$ \\
\hline
\end{tabular}

I. Injektion von $50 \mathrm{mg}$ Atoxyl am 12. XI. 08, vormittags $10^{1 / 2}$ Uhr.

\begin{tabular}{|c|c|c|c|c|c|}
\hline 1 & 240 & 24 & 48,4 & 0,0116 & 50,87 \\
\hline 2 & 80 & 32 & - & kein As & - \\
\hline & 110 & 48. & - & , & - \\
\hline & 120 & 4 Tage & - & .8 & - \\
\hline
\end{tabular}

II. Injektion von $50 \mathrm{mg}$ Atoxyl am 16. XI.08, nachmittags $4^{1 / 8}$ Uhr.

\begin{tabular}{l|r|rc|c|c|c}
5 & 250 & 24 & 43,96 & 0,0108 & 47,36 \\
6 & 160 & 48 & & - & & \\
7 & 100 & 3 & Tage & - & $0,000 \check{5}$ & 2,19 \\
8 & 120 & 8 & $\searrow$ & - & & \\
9 & 90 & 9 & $*$ & - & kein As & -
\end{tabular}

III. Injektion von $100 \mathrm{mg}$ Atoxyl am 24. XI. 08, nachmittags 4 Uhr.

\begin{tabular}{|c|c|c|c|}
\hline \begin{tabular}{l|l}
10 & 184
\end{tabular} & $1 \mathrm{Tag}$ & 92,69 & 1) \\
\hline 174 & 2 Tage & 3,14 & 00905 \\
\hline $20 \check{~}$ & $3>$ & - & \\
\hline 470 & 7 & - & \\
\hline 275 & 8 & - & $\begin{array}{l}\text { Spur Arsen } \\
\text { Kot: kein As }\end{array}$ \\
\hline
\end{tabular}

98,25

IV. Injektion von $300 \mathrm{mg}$ Atoxyl am 3.XII. 09, vormittags $11 \mathrm{Uhr}$. (Gewicht des Tieres 1,650 kg.)

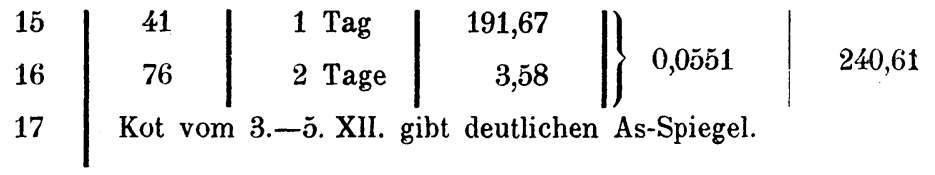


Tabelle V.

Katze von $1,760 \mathrm{~kg}$.

\begin{tabular}{c|c|c|c|c|c}
\hline \hline Nr. & $\begin{array}{c}\text { Harn- } \\
\text { menge } \\
\text { in ccm }\end{array}$ & $\begin{array}{c}\text { Stunden. } \\
\text { bezw. Tage } \\
\text { nach der } \\
\text { Injektion }\end{array}$ & $\begin{array}{c}\text { Ausgeschie- } \\
\text { denes Atoxyl } \\
\text { in mg }\end{array}$ & in g As & $\begin{array}{c}\text { entspr. Atoxyl } \\
\text { in mg }\end{array}$ \\
\hline
\end{tabular}

I. Injektion von $50 \mathrm{mg}$ Atoxyl am 15. XII. 08, vormittags $9^{1 / 2}$ Uhr.

\begin{tabular}{l|l|l|l|}
1 & 23 & $21 / 4$ & 32,84 \\
2 & 27 & $10^{3} / 4$ & 13,62 \\
3 & 23 & 24 & -
\end{tabular}

II. Injektion von $50 \mathrm{mg}$ Atoxyl am 16. XII. 08, vormittags $10.45 \mathrm{Uhr}$.

\begin{tabular}{l|l|l|l||c|c}
4 & 58 & 1 & 41,11 & & $\begin{array}{c}\text { Harn bis } \\
\text { 5. I. 09 }\end{array}$ \\
0,0421 & 184,65
\end{tabular}

III. Injektion von $50 \mathrm{mg}$ Atoxyl am 17. XII. 08, nachmittags 4 Uhr.

\begin{tabular}{l|r|c|c|}
6 & 100 & 24 & 37,97 \\
7 & 51 & 25 & -
\end{tabular}

IV. Injektion von $50 \mathrm{mg}$ Atoxyl am 18. XII, nachmittags $6 \mathrm{Uhr}$.

\begin{tabular}{l|l|l|c|}
8 & 118 & 24 & 26,05 \\
9 & 132 & 48 & -
\end{tabular}

Der Harn und Kot des ganzen Versuches wurde bis 5. I. 09 gesammelt und auf Gesamtarsen untersucht.

Harn und Kot vom 6. I. 09 waren arsenfrei.

Ergebnis : Injiziert $200 \mathrm{mg}$ A toxyl, als solches ausgeschieden $151,59 \mathrm{mg}$. Kot: 0,0021 g As, entsprechend 9,21 mg Atoxyl.

Tabelle VI.

Katze von $2,340 \mathrm{~kg}$, erhält vom 12. I. bis 17 . I. 09 täglich $50 \mathrm{mg}$ Atoxyl.

\begin{tabular}{c|c|c|c|c}
\hline \hline Nr. & Tagesharn & $\begin{array}{c}\text { Ausgeschiedenes } \\
\text { Atoxyl } \\
\text { in mg }\end{array}$ & \multicolumn{2}{|c}{ Gesamtarsen } \\
\cline { 4 - 5 } & in ccm & in m As & $\begin{array}{c}\text { entspr. Atoxyl } \\
\text { in mg }\end{array}$ \\
\hline 1 & 150 & 45,56 & & \\
2 & 130 & 45,43 & & \\
3 & 110 & 39,40 & & \\
4 & 65 & 34,45 & 0,0619 & 271,44 \\
5 & 60 & 41,88 & & \\
6 & 70 & 46,20 & & \\
7 & Kot & - & 0,0038 & 16,75
\end{tabular}


Tabelle VII.

Hund von $5,5 \mathrm{~kg}$ erhält vom 14. I. bis 21. I. 09 täglich Atoxyl.

\begin{tabular}{c|c|c|c|c|c|c}
\hline \hline Nr. & $\begin{array}{c}\text { Injiziertes } \\
\text { Atoxyl } \\
\text { in mg }\end{array}$ & $\begin{array}{c}\text { Tagesharn } \\
\text { in ccm }\end{array}$ & $\begin{array}{c}\text { Ausgeschie- } \\
\text { denes Atoxyl } \\
\text { in mg }\end{array}$ & in g As & $\begin{array}{c}\text { Gesamtarsen } \\
\text { in mg }\end{array}$ \\
\hline 1 & 50 & 350 & 40,50 & & \\
2 & 50 & 300 & 38,55 & & \\
3 & 100 & 430 & 82,13 & & \\
4 & 100 & 610 & 91,19 & & \\
5 & 100 & 600 & 72,00 & 0,1482 & 649,12 \\
6 & 100 & 710 & 85,20 & & \\
7 & 100 & 750 & 79,42 & & \\
8 & 100 & 600 & 90,00 & & & \\
9 & Kot & - & - & 0,0032 & 14,36
\end{tabular}

Tabelle VIII.

Mensch.

\begin{tabular}{l|c|c|c|c}
\hline \hline $\mathrm{Nr}$. & Harnmenge & $\begin{array}{c}\text { Stunden } \\
\text { nach der } \\
\text { Injektion }\end{array}$ & $\begin{array}{c}\text { Ausgeschiedenes } \\
\text { Atoxyl } \\
\text { in } \mathrm{mg}\end{array}$ & $\begin{array}{c}\text { Total ausge- } \\
\text { schiedenesAtoxyl } \\
\text { in } \mathrm{mg}\end{array}$ \\
\hline
\end{tabular}

I. Injektion von $50 \mathrm{mg}$ Atoxyl am 20. I. 09, vormittags $9 \mathrm{Uhr}$.

\begin{tabular}{|c|c|c|c|c|}
\hline 1 & 37 & bis 3 & 5,29 & \\
\hline 2 & 45 & $>6$ & 4,72 & 41,92 \\
\hline 3 & 405 & * 9 & 31,91 & \\
\hline 4 & 65 & $\gg 12$ & Spuren & - \\
\hline & 725 & $>24$ & - & - \\
\hline
\end{tabular}

II. Injektion von $50 \mathrm{mg}$ Atoxyl am 21. I. 09, vormittags $9 \mathrm{Uhr}$.

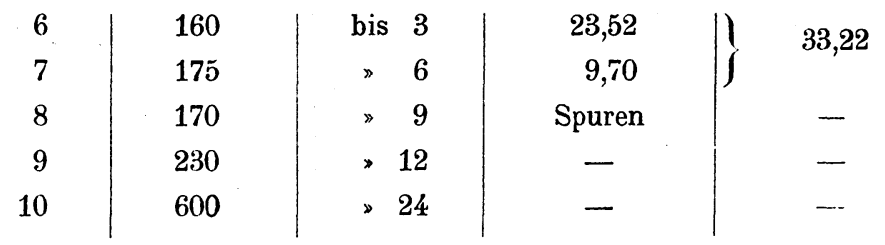

Ergebnis: Injiziert $100 \mathrm{mg}$ Atoxyl, als solches ausgeschieden $75,14 \mathrm{mg}$. Gesamt-As-Bestimmung 0,0191 g As, entsprechend 83,77 mg Atoxyl. 
Aus den Tabellen geht hervor, daß die Atoxylausscheidung bei den Kaninchen bereits nach 5-6 Stunden und beim Menschen nach 9 Stunden beendet ist. In einzelnen Fällen verläßt das Atoxyl innerhalb dieser Zeit nahezu quantitativ als solches den Organismus, und es wurden im Harn bis zu $96 \%$ des zugeführten Atoxyls wiedergefunden. Meist bewegen sich die Ausscheidungswerte von 50-90\%. Vor allem ergibt sich die bemerkenswerte Tatsache, daß das eingeführte Atoxyl im Körper eine Umwandlung erfährt, wie der Vergleich der ausgeschiedenen Atoxylmengen mit dem Gesamtarsen erkennen läßt.

Einen Beweis für die Brauchbarkeit der Methode und für die Annahme, daß der ausgeschiedene, die $\alpha$-Naphtholreaktion gebende Körper nur Atoxyl oder ein ihm nahestehender Körper sein kann, welcher noch die Amido- und Arsengruppe enthält, lieferten die Versuche, in denen das Atoxyl fast quantitativ wieder ausgeschieden wird (s. z. B. Tabelle IV, 1. Injektion), indem hier die Kongruenz des Gesamtarsens und des im Atoxyl ausgeschiedenen Arsens sehr deutlich zutage tritt.

Die Ausscheidung einer nicht als Atoxyl wiederkehrenden Arsenverbindung kann auch - und darin stimmen unsere Versuche völlig mit den früheren Autoren überein - noch mehrere Tage fortdauern. Allerdings wurden dann fast immer nur noch Spuren von Arsen gefunden.

Von Interesse war es, besonders bei den Kaninchen, die quantitativen Ausscheidungsverhältnisse mit dem klinischen Verhalten der Tiere zu vergleichen. Kaninchen B z. B., das bei den ersten 3 Injektionen das injizierte Gift nahezu restlos sofort wieder ausschied, zeigte während dieser Versuchsperiode keine Spur von Intoxikationserscheinungen, auch keinen Gewichtsverlust. Erst als bei der 4. Injektion von den eingeführten $300 \mathrm{mg}$ Atoxyl nur $240 \mathrm{mg}$ wieder ausgeschieden wurden, ging das Tier akut unter Krämpfen zugrunde. Anders verhielt sich Kaninchen A. Hier wurde von vornherein eine größere Menge Atoxyl im Körper zurückbehalten, und als Folge dieser Retention stellte sich zunehmende Abnahme des Körpergewichts ein.

Während wir bei den Kaninchen das Hauptaugenmerk 
auf die zeitlichen Ausscheidungsverhältnisse im Harn legten und deshalb den Kot nicht bei allen Versuchsperioden berücksichtigten, bestimmten wir bei den anderen Tieren stets auch den Arsengehalt der Faeces und der Organe. Es zeigte sich dabei, daß beim Hund von $700 \mathrm{mg}$ eingeführtem Atoxyl im Kot eine Menge Gesamtarsen zu finden war, die $14,36 \mathrm{mg}$ Atoxyl entsprechen, während bei der Katze von $200 \mathrm{mg}$ Atoxyl 9,21 mg durch den Darm ausgeschieden wurde.

Wenn auch zugegeben werden muß, daß die Ausscheidung des Atoxyls außer im Harn und Kot auch auf anderem Wege erfolgen kann, so zeigen die minimalen Arsenmengen, die Lockemann und Pauke ${ }^{1}$ ) in den Haaren fanden, daß diese Spuren gut vernachlässigt werden können, um so mehr, als Rabow und Stryzowski ${ }^{2}$ ) bei 10 atoxylbehandelten Negern selbst nach Gesamtmengen von 3-8 g Atoxyl kein As in den Haaren nachweisen konnten.

Im folgenden sei noch kurz die Berechtigung unserer Annahme begründet, daß der im Harn atoxylvergifteter Tiere auftretende und die $\alpha$-Naphtholreaktion gebende Körper in der Tat unzersetztes Atoxyl ist. Das uns von Herrn Geh.-Rat EhrlichFrankfurt a.M. gütigst zur Verfügung gestellte Reduktionsprodukt des Atoxyls, das p-Amidophenylarsinoxyd, $\mathrm{NH}_{2} \cdot \mathrm{C}_{6} \mathrm{H}_{4} \mathrm{AsO}_{2}$, gibt mit $\alpha$-Naphthol einen Azofarbstoff von derselben intensiv roten Farbe, wie ihn das Atoxyl bildet. Es wäre nun theoretisch denkbar, daß wir es auch im Harn unserer Tiere ganz oder teilweise mit dem Reduktionsprodukt des Atoxyls zu tun hatten. Jedoch dürfte das in Wirklichkeit nicht der Fall sein, wie das aus folgenden Versuchen hervorgeht. Wir konnten uns, wie Ehrlich selbst, von der außerordentlichen Giftigkeit des Reduktionsproduktes an Kaninchen überzeugen. Bei einer subcutanen Injektion von $20 \mathrm{mg}$ trat der Tod nach 2 Stunden ein, und nach Einverleibung von $10 \mathrm{mg}$ starb das Tier nach $1{ }^{1 / 2}$ Tagen. Im Harn beider Tiere entstand mit $\alpha$-Naphthol kein roter Farbst off. Der Harn des zweiten Tieres, den wir im Marshschen

1) Lockemann und Pauke, D. med. Woch., 1908, Nr. 34, S. 1460.

$\left.{ }^{2}\right)$ Rabow und Stryzowski, Therap. Monatshefte, 1908, April, S. 197. 
Apparat auf As untersuchten, war völlig As-frei. Die Giftigkeit der Substanz ging also mit ihrer Retention im Organismus parallel.

Nach alledem war anzunehmen, daß der Harn atoxylvergifteter Tiere, falls das Atoxyl in der Form des Reduktionsproduktes ausgeschieden wird, auf andere Tiere sehr erheblich giftig wirken mußte. Wir injizierten deshalb einem Kaninchen $200 \mathrm{mg}$ Atoxyl; der ausgeschiedene Harn wurde auf dem Wasserbade eingedampft und der Rückstand zur Trennung von anorganischem As mit heißem Methylalkohol extrahiert (vgl. S. 277). Der alkoholische Extrakt wurde bei ca. $30-40^{\circ}$ eingedampft und in wenig Wasser aufgenommen. In der Flüssigkeit fanden sich 159,6 mg Atoxyl. Hiervon wurden einem Kaninchen 26,6 mg und einem anderen 104,4 mg wirksamer Substanz subcutan injiziert. Beide Tiere blieben am Leben und zeigten keine wesentlichen Intoxikationserscheinungen. Daraus geht unzweideutig hervor, daß keine nennenswerten Mengen des Reduktionsproduktes im Harn erscheinen und daß in der Tat Atoxyl als solches zur Ausscheidung gelangt.

Diese Befunde stimmen gut mit denen Heffters ${ }^{1}$ ) überein, der nach der subcutanen Injektion von kakodylsaurem $\mathrm{Na}$ stets Kakodylsäure und nicht das Reduktionsprodukt Kakodyl im Harn nachweisen konnte.

Bevor wir dieses Kapitel verlassen, scheint es uns noch notwendig, auf die Arbeit von Lockemann und Pauke ${ }^{2}$ ) einzugehen, die als einzige Autoren bisher versuchten, das Atoxyl quantitativ zu bestimmen. Ihre Methode der Wägung des Azofarbstoffs ist schon deshalb nicht brauchbar, weil der $\beta$-Naphthylaminniederschlag bereits, wie sie selbst angeben, in atoxylfreiem Harn in kleinen Mengen entsteht. Ferner fanden sie schon häufig bis $12 \%$ zu wenig Atoxyl bei den zur Kontrolle angestellten Bestimmungen in reinen Atoxyllösungen; die Verfasser glauben dieses mangelhafte Resultat auf Beimengung von Nebenprodukten des Atoxyls beziehen zu müssen; unsere Versuche haben uns gezeigt, daß dieser Schluß ungerechtfertigt und daß das in den Handel gebrachte Atoxyl in der Tat rein

') Heffter, Arch. f. exp. Path. u. Pharm., 1901, Bd. XLVI, S. 230. 2) 1. c. 
ist. Die Resultate fielen aber noch schlechter aus, als sie den Atoxylgehalt im Harn bestimmten, "da darin meistens in wechselnder Menge Bestandteile enthalten sind, welche mit dieser Behandlungsweise mitausgefällt werden und daher die Exaktheit des Nachweises beeinträchtigt wird». Es würde zu weit führen, uns in eine Kritik von Einzelheiten der Untersuchungen einzulassen; teilweise wurden unsere Einwände auch schon von Blumenthal') in seiner Entgegnung hervorgehoben.

\section{B. Verhalten im Tierkörper.}

Wir untersuchten weiter den Gehalt des Blutes und einzelner Organe an Atoxyl. Das Verhalten des Atoxyls im Blute mußte schon deshalb interessieren, weil es ja seine hauptsächlichste therapeutische, d. h. trypanozide Wirkung im Blute entfaltet. Das morphologische Verhalten der einzelnen Blutbestandteile blieb bei diesen Versuchen unberücksichtigt; es lag uns nur daran, festzustellen, wie sich das Gift im Blute selbst verteilt und wie lange es sich im Kreislauf nachweisen läßt. $\mathrm{Zu}$ diesen Versuchen benutzten wir Kaninchen, denen wir das Atoxyl intravenös injizierten, um über die wirklich in das Blut gelangten Giftmengen einen sicheren Anhalt $\mathrm{zu}$ haben.

Es seien nun im folgenden einige derartige Versuche wiedergegeben. Die beiden ersten Versuche dienten uns mehr zur allgemeinen Orientierung, stimmen aber mit den übrigen in den charakteristischen Befunden gut überein, so daß wir sie hier beifügen.

\section{Versuch I.}

Kaninchen von $1,560 \mathrm{~kg}$ erhält intravenös $200 \mathrm{mg}$ Atoxyl. Nach 15 Minuten wird das Tier aus der Carotis verblutet und mit warmer physiologischer Kochsalzlösung durchspült.

Blut \{ Serum: 16,59 mg Atoxyl, $58 \mathrm{ccm}$ Blutkuchen gibt keinen As-Spiegel.

Durchspülungsflüssigkeit: Spuren Atoxyl.

\section{Versuch II.}

Kaninchen von $1,665 \mathrm{~kg}$ erhält intravenös $200 \mathrm{mg}$ Atoxyl. Nach 10 Minuten wird das Tier aus der Carotis verblutet und wie oben durchspült.

1) Blumenthal, Deutsche med. Wochenschr., 1908, Nr. 52, S. 2266. 
Über das Verhalten des Atoxyls im Organismus.

\section{Blut Serum: $14,85 \mathrm{mg}$ Atoxyl, $60 \mathrm{ccm}$ Blutkuchen: Spur As.}

Durchspülungsflüssigkeit: $8,28 \mathrm{mg}$ Atoxyl.

Harn enthält reichlich Atoxyl.

\section{Versuch III.}

Kaninchen von $1,577 \mathrm{~kg}$ erhält intravenös $500 \mathrm{mg}$ Atoxyl. Nach 5 Minuten verblutet und durchspült.

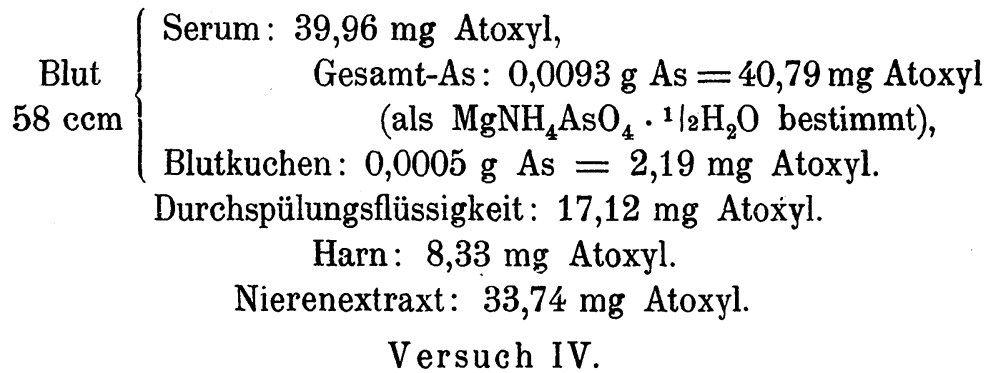

Kaninchen von $3,020 \mathrm{~kg}$ erhält intravenös $1 \mathrm{~g}$ Atoxyl. Nach $2^{1 / 2}$ Stunden verblutet und durchspült. Blut wird defibriniert.

Blut
$70 \mathrm{ccm}$$\left\{\begin{array}{c}\begin{array}{r}\text { Serum: } 2,14 \mathrm{mg} \text { Atoxyl, } \\ \text { Gesamtarsen: 0,0009 g As, entspr. 3,94mg Atoxyl } \\ \text { (Wägung des As-Spiegels). }\end{array} \\ \text { Blutkörper }\left\{\begin{array}{l}\text { ungewaschen: Schwacher As-Spiegel, } \\ \text { gewaschen: Spur As-Spiegel. }\end{array}\right. \\ \text { Fibrin: Kein As-Spiegel. }\end{array}\right.$

Durchspülungsflüssigkeit: Kein Atoxyl, ziemlich starker As-Spiegel.

\section{Harn $\{374,85 \mathrm{mg}$ Atoxyl. Nierenextrakt: Spur Atoxyl.}

$45 \mathrm{ccm}$ Gesamtarsen: 0,0943 g As, entspr. 413,59 mg Atoxyl.

\section{Augen: Kein As.}

Überblicken wir diese Versuchsreihe, so ist vor allem das eine evident, daß das Atoxyl im Serum gelöst den Körper durchkreist, während die geformten Elemente offenbar nur eine äußerst geringe Affinität zu dem Gifte besitzen. Bei Versuch III konnten allerdings immerhin nennenswertere As-Mengen im Blutkuchen nachgewiesen werden, doch ist es wahrscheinlich, daß ein Teil des darin gefundenen As auf noch anhaftendes 
Serum zurückzuführen ist. Wir gebrauchten deshalb in Versuch IV die Vorsicht, die Blutkörperchen mit physiol. NaClLösung zu waschen, fanden allerdings keinen wesentlichen Unterschied im As-Gehalt zwischen ungewaschenen und gewaschenen Blutelementen; beidesmal entstand geringer As-Spiegel. Auch das Fibrin scheint kein As zu fixieren.

Die Atoxylwerte, die wir im Serum gefunden haben, können nicht den Anspruch erheben, den wahren Gehalt des Gesamtblutes an Atoxyl wiederzugeben, da man beim Entbluten nur etwa bis $1 / 2$ der Gesamtblutmenge erhält, doch geben sie uns gut brauchbare und miteinander vergleichbare relative Werte. Um das im Gefäßsystem noch vorhandene Blut nach Möglichkeit noch der Untersuchung zugänglich zu machen, spülten wir mit physiol. NaCl-Lösung nach und fanden bei den kurzdauernden Versuchen (I-III) noch erhebliche Atoxylmengen. 21/2 Stunden nach der Injektion (Vers. IV) war in der Durchspülungsflüssigkeit kein Atoxyl mehr nachweisbar. Während in Vers. I-III nach 5, 10 und 15 Minuten noch ansehnliche Atoxyl-Quantitäten in Serum und Durchspülungsflüssigkeit zu finden waren, zeigt Versuch IV nach $21 / 2$ Stunden nur einen minimalen Atoxylgehalt und läßt weiter unzweideutig erkennen, daß das Atoxyl bereits eine Umwandlung erfahren hat, wie das aus dem Vergleich der Gesamt-As-Bestimmung mit den gefundenen Atoxylmengen teilweise hervorgeht. Dieser quantitative Unterschied ist trotz der Kleinheit der Mengen bei der Genauigkeit der Methode (Wägung des As-Spiegels) nicht als innerhalb der Fehlergrenzen liegend anzusehen. Besonders beweisend für die Umwandlung ist der positive As-Befund in der Durchspülungsflüssigkeit bei gänzlichem Ausbleiben der Atoxylreaktion.

Die beigefügten Nieren- und Harnanalysen zeigen, wie erstaunlich große Atoxylmengen bereits 5 Minuten nach der Injektion wieder aus dem Organismus ausgeschieden werden. Es ist daher auch verständlich, daß bei der Sektion des Kaninchens von Versuch III bereits streifige Blutungen in der Niere nachzuweisen waren.

Das Atoxyl verschwindet nach den mitgeteilten Befunden weit langsamer aus dem Blut als arsenigsaures Salz, von dem 
nach Morishima ${ }^{1}$ ) schon wenige Minuten nach der intravenösen Injektion nur noch Spuren im Aderlaßblut nachweisbar sind. Obgleich schon in dẹ ersten Zeit nach der Einverleibung des Atoxyls die im Harn ausgeschiedene Menge sehr beträchtlich sein kann, findet man $2^{1 / 2}$ Stunden nach der intravenösen Injektion großer Mengen noch mehrere Miligramme Atoxyl im Blut. Ein Teil der in den Geweben noch zurückgebliebenen Menge wird in relativ kurzer Zeit (2 $1 / 2$ Stunden) in eine andere Form umgewandelt, so daß die Gesamt-As-Bestimmung im Blut sowie in der Durchspülungsflüssigkeit höhere Werte ergibt, als dem Atoxyl entspricht.

Der durch längere Zeit bestehende Gehalt des Blutserums an Atoxyl ist für die Erklärung der Wirkung den Trypanosomen gegenüber von Interesse. Die Trypanosomen schwimmen frei im Blutserum, die Malariaplasmodien, welche in den Blutkörperchen eingeschlossen sind, scheinen gegen Atoxyl resistenter zu sein. Dies erklärt sich vielleicht durch das mangelnde Eindringen des Atoxyls in die Blutkörperchen.

Bei der Syphilis, wo das Atoxyl ebenfalls allerdings mit wechselndem Erfolg angewandt wird, kommen die spezifischen Spirochaeten hie und da schubweise ins Blut und bewegen sich zur Hauptsache frei in ihm. Ein positiver Heilerfolg durch Atoxyl könnte hier so erklärt werden, daß gerade zur Zeit der Atoxylverabreichung das Blut mit Spirochaeten überschwemmt ist. Durch die angedeuteten Verteilungsverhältnisse wird also der Weg zu den verschiedenen Protozoen dem Atoxyl mehr oder weniger geebnet. Hiervon abgesehen können selbstverständlich bestimmte einzellige Wesen, wie die Trypanosomen, noch besonders starke Affinitäten für das Gift besitzen.

Es ist nach den obigen Versuchen nicht wunderbar, daß bereits früher von einzelnen Autoren (Blumenthal, Wedemann ${ }^{2}$ )) qualitativ As im Blut nachgewiesen werden konnte; ebenso wenig ist allerdings erstaunlich, daß Blumenthal 3 Stunden nach der Injektion von 0,5 g Atoxyl bei der Übertragung des Blutes dieses Versuchstieres auf ein anderes Kaninchen keine Krankheitserscheinungen auftreten sah; wie wir

1) Morishima, Arch. intern. de Pharmacodyn. et de Thérap., Bd. VII, 1900.

2) Wedemann, Arb. a. d. Kais. Ges.-Amt, 1908, Bd. XXVIII, S. 585. 
jetzt wissen, wird es sich bei diesem Versuch nur um wenige Zehntelmilligramme As bezw. Atoxyl gehandelt haben. ${ }^{1}$ )

Über den As-Gehalt verschiedener Organe nach Atoxylinjektionen ist bereits von mehreren Autoren berichtet worden; so wurde As nachgewiesen in der Leber (Blumenthal und Jakoby, Groner u. Seligmann, Wedemann), in der Niere (Wedemann), im Gehirn und in der Knochensubstanz (Blumenthal und Jakoby), doch handelt es sich bei diesen Untersuchungen nur um qualitativen Nachweis.

Uns schien es bei unseren Untersuchungen besonders wichtig, die in den Organen aufgespeicherten As-Mengen quantitativ zu bestimmen und sie mit den in Harn und Kot ausgeschiedenen Mengen zu vergleichen, um auf diese Weise einen besseren Einblick in den Atoxylhaushalt zu gewinnen. Wir berücksichtigten dabei allerdings nur die inneren Organe (Leber, Niere, Milz, Darm, Lunge, Her $/$ ) und verarbeiteten diese gemeinsam; vernachlässigt wurden Muskel- und Knochensystem, sowie Haut und Haare.

Aus klinischen und experimentellen Erfahrungen ist es bekannt, daß die Augen der verschiedensten Tiere von dem Atoxyl in erheblichem Maße affiziert werden; ferner wissen wir, daß das Zentralnervensystem der Katze in eigenartiger Weise auf Atoxyl reagiert; es war daher als weitere Frage von Interesse, nachzusehen, ob sich in diesen genannten Organen As nachweisen läßt.

Zwischen der letzten Atoxylinjektion und dem Tod der Tiere lagen stets mehrere Tage dazwischen.

In der folgenden Tabelle IX finden wir die Resultate dieser Untersuchungen zusammengestellt.

Aus der tabellarischen Übersicht geht hervor, daß beim

1) Anmerkung bei der Korrektur: Auf Grund unserer Befunde über das Verhalten des Atoxyls im Blut sind die Resultate von Uhlenhuth und Woithe, deren letzte Arbeit (Arb. a. d. Kais. Ges.-Amt, 1908, Bd. XXIX) uns erst jetzt zu Gesicht kam, verständlich, daß prophylaktisch gegebenes Atoxyl den Ausbruch der Dourine nicht verhindern kann, daß aber bei möglichst frühzeitiger Behandlung der bereits bestehenden Dourine mit Atoxyl gute Heilungen erzielt werden. 
Über das Verhalten des Atoxyls im Organismns.

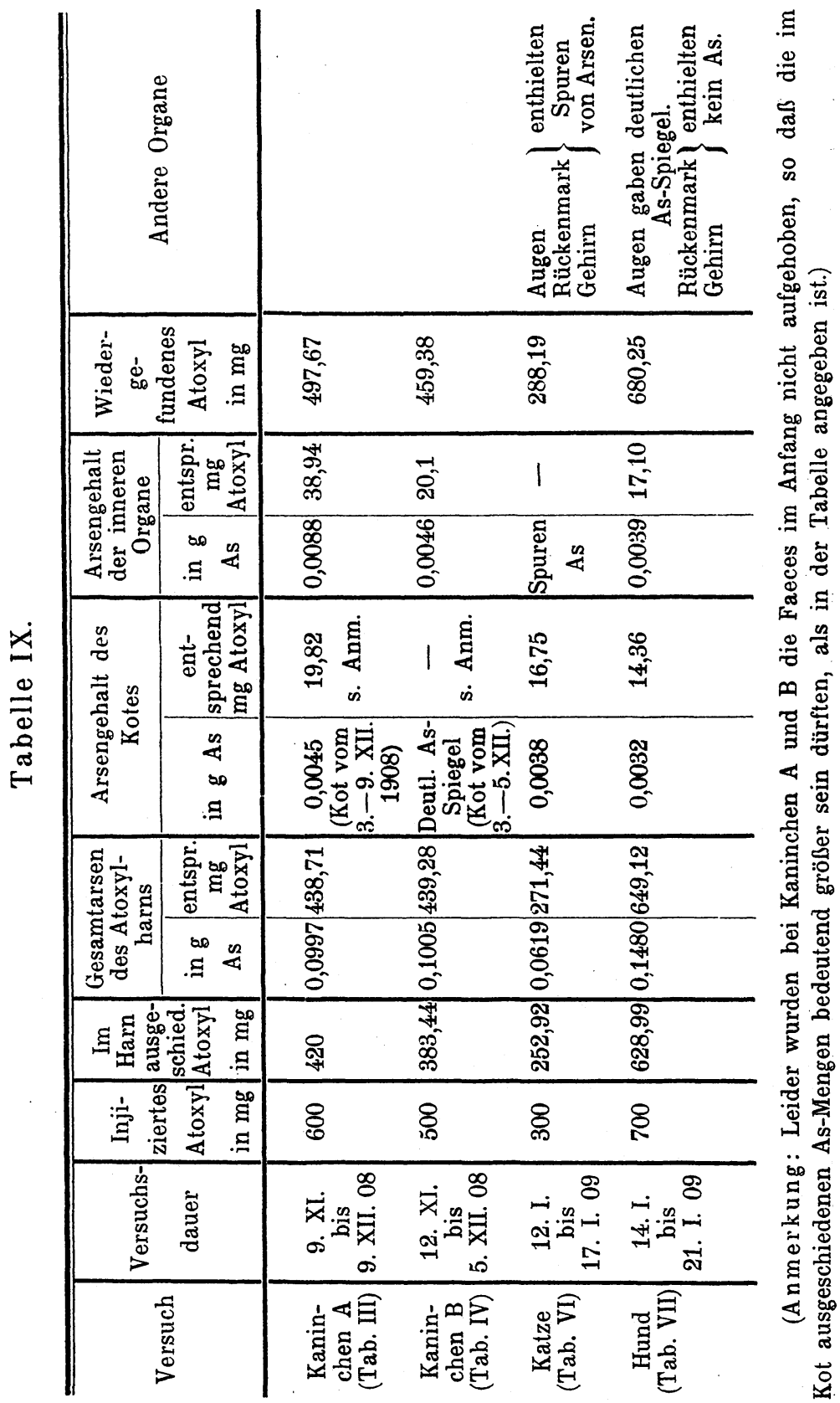


Kaninchen und Hund ziemlich ansehnliche As-Mengen in den Organen gefunden wurden $(2-6 \%$ des eingeführten Atoxyls), während in den inneren Organen der Katze sich nur Spuren As fanden. Dagegen waren Rückenmark und Gehirn der Katze arsenhaltig, Zentralnervensystem des Hundes dagegen arsenfrei. Das Vorwiegen des As im Nervensystem der Katze einerseits und den inneren Organen des Hundes anderseits steht in gutem Einklang mit den klinischen und pathologisch-anatomischen Befunden bei diesen beiden Tierarten.

Von besonderem Interesse war uns der positive AsBefund in den Augen von Katze und Hund. Auch hier entspricht der chemische Befund den anatomischen Veränderungen. Es entstand nun noch die Frage, ob auch bei Verabreichung von anorganischen As-Verbindungen As in den Bulbi nachweisbar ist. Wir injizierten deshalb einer Katze im Laufe einer Woche $150 \mathrm{mg}$ arsensaures $\mathrm{Na}$, die ihrem As-Gehalt nach den bei der Atoxylkatze verwandten Atoxyldosen entsprechen. Das Tier wurde schwer krank, litt an einer heftigen Conjunctivitis und Rhinitis, verlor stark an Gewicht und wies schließlich die für anorganische As-Intoxikation charakteristische periphere Lähmung der Extremitäten (im Unterschied zu der spastischen Lähmung nach Atoxylvergiftung) auf. Die Untersuchung der Augen auf As im Marshschen Apparat ergab negatives Resultat. Ebenso war in den Augen normaler Tiere kein As zu finden.

Es wird durch diesen chemischen Nachweis die auch schon aus klinischen und experimentellen Erfahrungen wahrscheinliche Annahme gestützt, daß es sich bei der Erkrankung der Augen und des Zentralnervensystems um eine Beeinflussung dieser Organe durch das Atoxyl selbst oder eventuell ein ihm nahestehendes Reduktionsprodukt und nicht durch abgespaltenes anorganisches As handelt.

Die Frage über die wirksame Komponente des Atoxyls steht, besonders was die therapeutische Beeinflussung der Trypanosomiasis anbetrifft, noch immer zur Diskussion. Während E hr- 
lich , 1) Levaditi, ${ }^{2}$ ) Friedberger ${ }^{3}$ ) u. a. die Ansicht vertreten, daß das Atoxyl im Körper reduziert wird und so oder in einer Eiweißverbindung (Levaditis "Trypanotoxyl») zur Wirkung kommt, neigt Blumenthal immer noch der Meinung zu, daß die Wirkung des Atoxyls auf abgespaltenem anorganischen As beruht. Blumenthal und Jacoby ${ }^{4}$ ) haben in ihrer neuesten Veröffentlichung das Für und Wider dieser Möglichkeiten besprochen, und können wir, um Wiederholungen zu vermeiden, auf diese Zusammenstellung verweisen.

Wir suchten der Frage, ob anorganisches As aus dem Atoxylmolekül abgespalten wird, näher zu kommen, indem wir den Harn atoxylvergifteter Kaninchen nach der von E. Salkowski ${ }^{j}$ ) angegebenen Methode auf anorganisches und organisches Arsen untersuchten, bedienten uns aber zur Trennung, da das Atoxyl in Äthylalkohol fast unlöslich ist, des Methylalkohols, in welchem Atoxyl sich leicht löst; dabei findet sich das anorganische Arsen in dem alkoholunlöslichen Rückstand. Die Untersuchung dieses Rückstandes ergab bei zwei Kaninchen nach einmaliger lnjektion von 500 resp. $200 \mathrm{mg}$ Atoxyl einen starken bezw. schwachen As-Spiegel, so daß demnach eine Abspaltung von As, in kleinen Mengen wenigstens, anzunehmen ist. Mit Hilfe dieser Methode dürfte wohl in diese Dinge noch weiteres Licht $\mathrm{zu}$ bringen sein.

Wir suchten ferner festzustellen, ob bei der Autolyse von Organen eine Zerstörung von zugefügtem Atoxyl stattfindet. $\mathrm{Zu}$ diesem $\mathrm{Zweck}$ wurden die betreffenden Organe mit physiologischer Kochsalzlösung zu einem Brei zerrieben und diesem Brei bestimmte Mengen Atoxyl zugefügt. Das Gemisch wurde sodann mit Toluol überschichtet, im Brutschrank bei $36-37^{\circ}$ aufbewahrt und nach verschieden langer Zeit auf Atoxyl und Arsen untersucht. Wir saugten den Organbrei

1) Ehrlich, Münch. med. Wochenschr., 1909, Nr. 5.

2) Levaditi und Yamamouchi, Soc. de la biolog., 1908, Bd.LXV, S. 23, und Levaditi, Bull. de la Soc. de pathol. exotique, 1909, S. 45.

3) Friedberger, Berl. klin. Wochenschr., 1908, Nr. 38, Sep.-Abdr.

4) Blumenthal und Jakoby, Biochem.Zeitschr., 1909, Bd.XVI, S. 20.

5) E. Salkowski, Biochem. Zeitschr., Bd. XIII, ๖. u. 6. H., S. 327. 


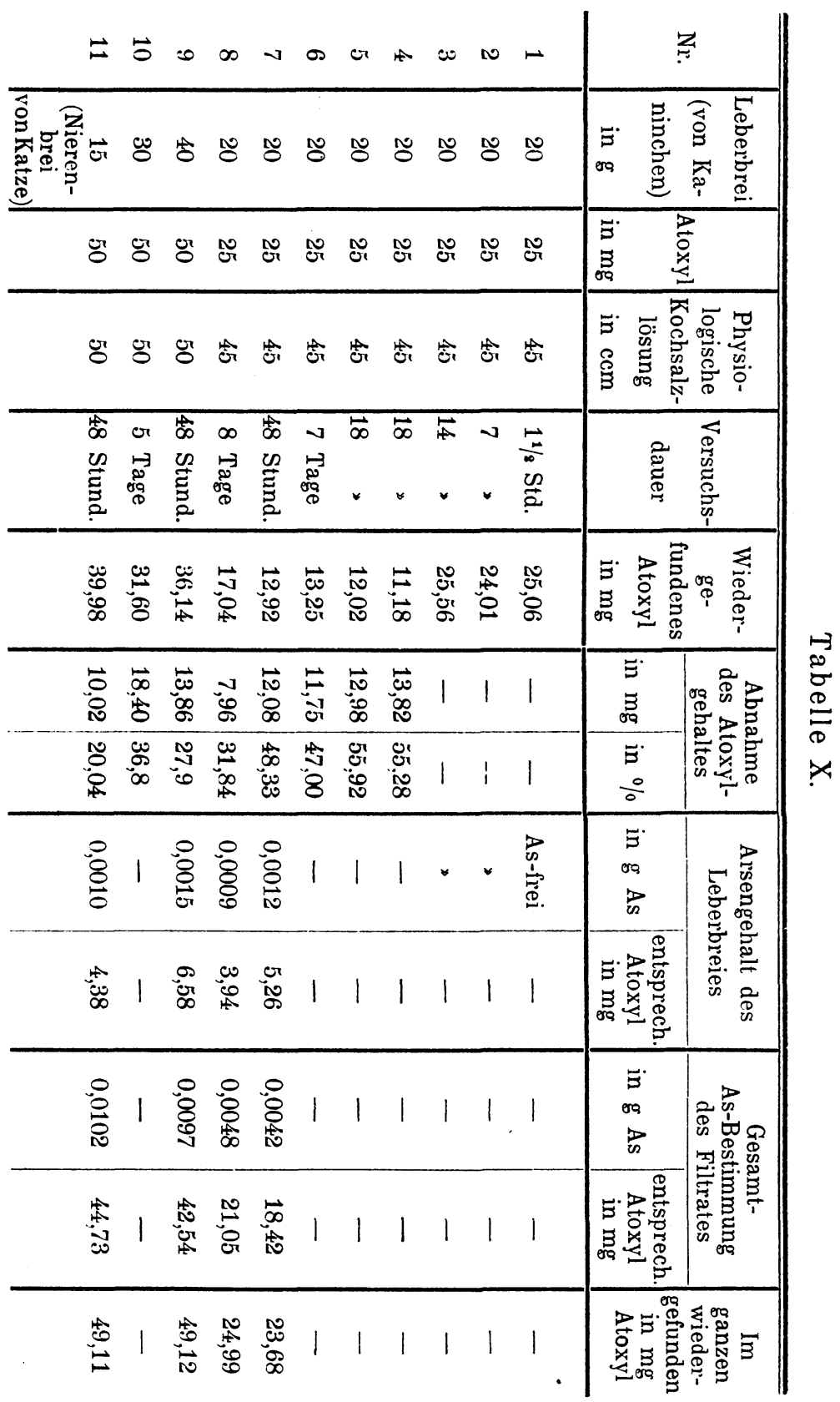


scharf $a b$, wuschen gründlich und so lange nach, bis das Waschwasser nicht mehr die $\alpha$-Naphtholreaktion gab, und bestimmten im Filtrat 1. den Atoxylgehalt und 2. den Gesamtarsengehalt sowie den Arsengehalt nach Marsh im Rückstand.

Aus vorstehender Tabelle $\mathrm{X}$ ergibt sich die interessante Tatsache, daß das Atoxyl in den ersten 14 Stunden unverändert im Filtrat wiederzufinden ist. Nach 18 stündiger Autolyse konnten wir dagegen eine beträchtliche Abnahme des Atoxylgehaltes feststellen. Die Abnahme war bei kleinen Atoxylmengen prozentual größer als bei höheren Dosen. Auffallend war, daß nach mehrtägiger Autolyse eine vermehrte Abnahme nicht erfolgt war. Wir lassen es dahingestellt, ob diese Hemmung einer weiteren Abnahme auf einer fermenttötenden Bildung von arseniger Säure beruht. ${ }^{1}$ )

Der Gesamtarsengehalt des Filtrates war stets um etwa 4-6 mg Atoxyl höher als der kolorimetrisch gefundene Atoxylwert. Ferner fanden wir dann auch stets Arsen im Organbrei. Aus alledem ergibt sich, daß das Atoxyl bei länger dauernder Autolyse eine erhebliche Zerstörung erleidet. Fraglich ist nun; in welcher Form das As abgespalten wird. Die Tatsache jedoch, daß der Organbrei nach dem Kochen mit Salzsäure nicht die $\alpha$-Naphtholreaktion gibt, und ferner die Unwahrscheinlichkeit, daß Anilin resp. p-Amidophenol sich gebildet hat, drängt zu der Annahme, daß es sich wohl um anorganisches Arsen handeln wird.

Zum Schluß versuchen wir einige Konsequenzen aus den beobachteten Tatsachen zu ziehen.

Da wir das eingeführte Atoxyl größtenteils unzersetzt im Harn wiederfinden, so liegt keine Schwierigkeit in der Annahme, daß es auch in unveränderter Form im Körper wirken kann. Jedenfalls ist es aber in dieser Form relativ ungiftig. Die Angriffspunkte des Atoxyls liegen sowohl in den Trypanosomen im Blute als auch in den Organen. Die große Menge Atoxyl, die im Anfange nach der Injektion im Blut zu

1) Vgl. Laqueur, Schriften der phys. ökon. Ges. zu Königsberg, Bd. XLVIII, 1907, Sep. Abdr. 
finden ist, läßt es verständlich erscheinen, daß hier und da bereits nach einer einzigen Atoxylinjektion Abtötung der Trypanosomen d. h. Heilung der Schlafkrankheit erzielt wurde, wie dies von Robert $\mathrm{Koch}^{1}$ ) berichtet wird.

Es findet ferner auch eine Bindung des Atoxyls durch Organzellen statt. Daß die Giftwirkung auf die Organe mit dieser Bindung parallel geht, beweisen die positiven As-Befunde in denjenigen Organen (Augen, Zentralnervensystem der Katze, innere Organe des Hundes), in denen klinisch und anatomisch die Veränderungen nach Atoxylvergiftung vornehmlich lokalisiert sind. ${ }^{2}$ ) Auch die Autolyseversuche unterstützen diese Ansicht und machen es außerdem wahrscheinlich, daß gerade in den Organen eine Umwandlung resp. Zerstörung eines Teils des Atoxyls stattfindet.

Bei der Zerstörung des Atoxyls spielt sicher, wie die Autolyseversuche und die Ergebnisse der Harnanalysen anzeigen, auch die Abspaltung von anorganischem Arsen eine Rolle. Damit scheinen die Spätsymptome bei längerer Behandlung im Zusammenhang zu stehen, denn die bei chronischer Atoxylvergiftung auftretenden Vergiftungssymptome sind zum Teil analog den Erscheinungen bei Arsenvergiftungen. Diese für Arsenvergiftung charakteristischen Erscheinungen (Conjunctivitis, Rhinitis, Pharyngitis, trophische Störungen an der Haut usw.) traten bei der akuten und subakuten Atoxylintoxikation niemals auf.

Es ist wahrscheinlich, daß das unzersetzt im Blute kreisende Atoxyl von den Zellen, die es besonders stark an sich reißen - sei es Organzellen, sei es Trypanosomen -, in das giftige Reduktionsprodukt umgewandelt wird. Auf Grund unserer eigenen Beobachtungen können wir diese Frage nicht entscheiden. Dagegen erscheint es uns sicher, daß wir es bei der Atoxylvergiftung mit zwei wirksamen Faktorenzu tun haben, dem Atoxyl resp. seinem Reduktionsprodukt einerseits und dem abgespalteten anorganischen As anderseits.

1) Deutsche med. Wochenschr., 1907, Nr. 46, S. 1889.

2) Für das Arsen wurde durch Slowtzoff (Hofmeisters Beitr., Bd. I, 1902, S. 281) nachgewiesen, daß nach Verfütterung von $\mathrm{As}_{2} \mathrm{O}_{3}$ eine sehr feste Bindung des As durch die Lebernucleine stattfindet. 